\title{
Freqüência da profilaxia mecânica para trombose venosa profunda em pacientes internados em uma unidade de emergência de Maceió
}

\author{
Frequency of mechanical prophylaxis for deep venous thrombosis in \\ patients admitted to an emergency room in Maceió, Brazil
}

\author{
Nathalia Leilane Berto Machado ${ }^{1}$, Ticiana Leal e Leite ${ }^{2}$, Guilherme Benjamin Brandão Pitta ${ }^{3}$
}

\section{Resumo}

Contexto: A trombose venosa profunda (TVP) é uma doença de ocorrência multidisciplinar e freqüente, incluindo as complicações relacionadas a ela, como o tromboembolismo pulmonar (TEP). Sendo a profilaxia mecânica um dos melhores (por seu baixo custo e eficácia comprovada) e mais simples meios para reduzir sua incidência, é de grande relevância que se pesquise sua utilização visando benefícios para o paciente e o serviço hospitalar.

Objetivo: Determinar a freqüência da utilização da profilaxia mecânica para TVP na Unidade de Emergência Dr. Armando Lages em Maceió (AL).

Métodos: Foi realizado um estudo transversal descritivo na unidade durante o período de 8 meses. A amostra foi calculada em 282 pacientes internados nas unidades de enfermarias das diversas especialidades. Os dados foram coletados em prontuários e mediante entrevista ao paciente. No prontuário de cada paciente foi pesquisada a utilização da profilaxia mecânica para TVP, além de diversos fatores para a estratificação do risco, segundo a Sociedade Brasileira de Angiologia e Cirurgia Vascular. O estudo estatístico foi realizado através do software SPSS, utilizando o teste qui-quadrado considerando o valor de $\mathrm{p}<0,05$.

Resultados: Foram analisados 282 pacientes, sendo 181 (64\%) homens e 101 (36\%) mulheres, com idade média de 54,1 anos. Quanto ao risco, $210(74,5 \%)$ foram classificados como alto risco, $56(19,8 \%)$ como moderado risco e $16(5,7 \%)$ como baixo risco. Do total de pacientes, $234(83 \%)$ não receberam profilaxia e $48(17 \%)$ receberam. Não houve diferença estatística entre os dados obtidos na pesquisa e os encontrados na literatura $(\mathrm{p}=0,065)$.

Conclusões: Apesar de ter sua eficácia comprovada e difundida, a profilaxia mecânica para TVP ainda não atinge níveis satisfatórios de utilização.

Palavras-chave: Trombose venosa, profilaxia, tromboembolismo.

\begin{abstract}
Background: Deep venous thrombosis (DVT) is a multidisciplinary and frequent disease, also including complications such as pulmonary thromboembolism. Mechanical prophylaxis is one of the best (due to its low cost and proven effectiveness) and simplest means to reduce its incidence; therefore, investigating its use as an attempt to enhance benefits to the patient and hospital service is of great importance.

Objective: To determine the frequency of mechanical prophylaxis for DVT at the Emergency Room Dr. Armando Lages in Maceió, Brazil.

Methods: A descriptive cross-sectional study at the emergency room was carried out for an 8 -month period. The sample was composed of 282 patients admitted to the nursing wards of various specialties. Data were collected from medical records and by patient interview. Each patient's medical record was searched for use of mechanical prophylaxis for DVT, in addition to several factors for risk stratification, according to the Brazilian Society of Angiology and Vascular Surgery. Statistical analysis was performed using SPSS software and chi-square test, considering $\mathrm{p}<0.05$.
\end{abstract}

Results: A total of 282 patients were analyzed, 181 (64\%) men and $101(36 \%)$ women, mean age of 54.1 years. Classification of risk was as follows: $210(74.5 \%)$ were classified as high risk, $56(19.8 \%)$ as moderate risk, and $16(5.7 \%)$ as low risk. Of all patients, $234(83 \%)$ did not received prophylaxis and $48(17 \%)$ did. There was no statistical difference between the data obtained in the research and those found in the literature $(\mathrm{p}=$ 0.065).

Conclusions: Despite having its efficacy confirmed, mechanical prophylaxis for DVT has not reached satisfactory levels of use.

Keywords: Venous thrombosis, prophylaxis, thromboembolism.

1. Bolsista, Programa Institucional de Bolsas de Iniciação Científica. Acadêmica de Fisioterapia, Universidade Estadual de Ciências da Saúde de Alagoas (UNCISAL), Maceió, AL.

2. Fisioterapeuta, UNCISAL, Maceió, AL. Mestranda, Universidade Federal de São Paulo (UNIFESP), São Paulo, SP.

3. Doutor. Professor adjunto, Departamento de Cirurgia, UNCISAL, Maceió, AL. Cirurgião vascular, Unidade de Emergência Armando Lages, Maceió, AL. Hospital Escola José Carneiro, Maceió, AL.

Trabalho realizado na Universidade Estadual de Ciências da Saúde de Alagoas (UNCISAL), Maceió, AL, e apresentado na condição de tema livre oral no $37^{\circ}$ Congresso Brasileiro de Angiologia e Cirurgia Vascular (CBACV), realizado em 2007, e na condição de pôster no $4^{\circ}$ Congresso Internacional de Fisioterapia, realizado em setembro de 2008.

Apoio financeiro: Programa Institucional de Bolsas de Iniciação Científica (PIBIC), concedido pelo Conselho Nacional de Desenvolvimento Científico e Tecnológico (CNPq).

Não foram declarados conflitos de interesse associados à publicação deste artigo.

Artigo submetido em 24.08.08, aceito em 25.09.08.

J Vasc Bras. 2008;7(4):333-340.

Copyright@ 2008 by Sociedade Brasileira de Angiologia e de Cirurgia Vascular 


\section{Introdução}

A trombose venosa profunda (TVP) é uma doença de ocorrência multidisciplinar caracterizada pela formação de trombos de forma oclusiva total ou parcial em veias do sistema venoso profundo $^{1}$, bastante freqüente, principalmente como complicações de outras afecções cirúrgicas e clínicas. No entanto, também pode ocorrer espontaneamente em pessoas aparentemente hígidas. $\mathrm{O}$ quadro clínico local depende, em grande parte, da extensão da área acometida e das veias atingidas, podendo acompanhar-se de manifestações sistêmicas e complicações como a embolia pulmonar ${ }^{2}$.

O desenvolvimento do tromboembolismo venoso depende da alteração em um ou mais fatores da tríade descrita por Virchow em 1856, que considera as alterações do fluxo sanguíneo (estase venosa), de componentes sanguíneos (hipercoagulabilidade) e da parede vascular (lesão endotelial) como responsáveis pelo processo trombótico ${ }^{2}$. Porém, desde essa época, a estase passou a ser considerada como o principal fator predisponente à trombose venosa. A relação entre a estase e a trombose dos membros inferiores surgiu da associação encontrada entre tempo de repouso e incidência de TVP em trabalhos clínicos e anatomopatológicos. Tanto a diminuição da velocidade de fluxo como a do volume do mesmo fazem parte do termo estase sanguínea e são suspeitas de desempenharem um importante papel no desenvolvimento do processo trombótico ${ }^{2}$.

A TVP é a terceira causa mortis de doenças cardiovasculares nos Estados Unidos ${ }^{3}$. Admite-se que a incidência anual do tromboembolismo venoso (TEV) diagnosticado é de um a dois episódios por 1.000 habitantes na população geral. Dados recentes indicam uma incidência de 500.000 casos anuais de TEV nos EUA, com aproximadamente 50.000 mortes por embolia pulmonar ${ }^{4}$.

Por ser a principal causa de tromboembolismo, é de extrema importância que se realize a profilaxia da TVP, pois a embolia pode ser a primeira manifestação clínica da doença e costuma ser fatal em $0,2 \%$ dos pacientes internados ${ }^{5}$.
Apesar da profilaxia para TVP ser aceita como uma estratégia bem estabelecida e eficaz, com recomendações detalhadas que devem ser empregadas em todas as classes de pacientes hospitalizados ${ }^{6,7}$ e de protocolos de prevenção da doença estarem à disposição de todos os profissionais da área da saúde, muitos não a utilizam rotineiramente ${ }^{8-10}$.

Algumas pesquisas mostraram uma baixa aderência à profilaxia contra os eventos trombóticos ou seu uso incorreto, quando realizada. No entanto, a prevenção adequada reduz em dois terços os casos de TVP e em um terço os casos de TEP, o que torna extremamente importante o conhecimento dos diferentes grupos de risco e seus meios de prevenção $0^{2,9,11}$.

Duas abordagens podem ser feitas para prevenir a trombose e o risco conseqüente de embolia pulmonar fatal: a profilaxia secundária que corresponde à detecção precoce e ao tratamento de TVP subclínica com o objetivo de prevenir um possível tromboembolismo; e a profilaxia primária que diz respeito à utilização de métodos físicos e/ou farmacológicos com finalidade de impedir ou minimizar a chance de um paciente desenvolver trombose ou embolia pulmonar.

A prevenção primária é preferida na maioria das circunstâncias clínicas. A base de sua utilização está no caráter clinicamente silencioso da TVP em mais da metade dos casos, de sua relativamente alta incidência em pacientes hospitalizados e nas conseqüências eventualmente graves do diagnóstico não realizado ou realizado tardiamente ${ }^{12}$. A prevenção secundária deve ficar reservada aos pacientes nos quais a profilaxia primária é contra-indicada ou inefetiva ${ }^{13}$.

A profilaxia fisioterapêutica é mais comumente conhecida como mecânica ou física, pois alguns de seus métodos não são restritos à fisioterapia. Essa profilaxia atua no combate ao principal fator da tríade de Virchow (estase sanguínea) através de métodos que aumentam o retorno venoso, além de ser indicada em todas as estratificações de risco e em casos de predisposição à hemorragia pela profilaxia farmacológica ${ }^{14}$.

O objetivo deste estudo foi determinar a freqüência da utilização da profilaxia mecânica 
da TVP em unidade de emergência de Maceió com a hipótese de que a mesma é pouco utilizada segundo relatos na literatura.

\section{Material e métodos}

O presente estudo foi aprovado pelo comitê de ética em pesquisa da UNCISAL através do parecer $\mathrm{n}^{\circ} .657$ 12007, com a permissão da direção da Unidade de Emergência Dr. Armando Lages e foi conduzido de acordo com a resolução 196/96 do Conselho Nacional de Saúde.

Foi realizado um estudo transversal prospectivo descritivo, com a finalidade de determinar a freqüência de utilização da profilaxia mecânica para TVP na unidade de emergência durante o período de dezembro de 2007 a julho de 2008 .

O tamanho da amostra foi estimado em 282 pacientes, considerando a freqüência de profilaxia de $25 \%$ numa população definida como finita através de uma média do número de pacientes internados na unidade de emergência nos 3 últimos anos, com precisão absoluta de $5 \%$ e nível de significância de 5\%. Foi utilizada uma calculadora eletrônica disponível na Internet (http: //lia.uncisal.edu.br/ensino/pdf2/CTA_Proporcao_ finita.xls), na qual os dados foram inseridos.

A coleta de dados foi realizada através da análise dos prontuários hospitalares e através de entrevista aos sujeitos. Foram incluídos os pacientes internados nas unidades de enfermarias das diferentes especialidades da unidade de emergência no período da pesquisa. Os critérios de exclusão foram: pacientes menores de 18 anos e índios (pois segundo a resolução 196 do Conselho Nacional de Saúde, em comunidades culturalmente diferenciadas como indígenas deve-se contar com a anuência antecipada da comunidade através dos seus próprios líderes, além de não se apresentarem em número relevante no período do estudo).

Cada paciente foi avaliado e estratificado quanto ao risco para TVP, pesquisando-se fatores clínicos, cirúrgicos e medicamentosos, através de um protocolo de estudo recomendado pela Sociedade Brasileira de Angiologia e Cirurgia Vascular (SBACV). A Tabela 1 mostra os dados contidos no protocolo utilizado os quais foram pesquisados nos registros hospitalares. Concluído o preenchimento do protocolo de acordo com os dados disponíveis no prontuário de cada paciente, o mesmo era entrevistado (após esclarecimento e assinatura do termo de consentimento livre e esclarecido) para uma maior fidedignidade da classificação do risco. Os sujeitos foram estratificados em baixo, médio e alto risco, de acordo com as normas da SBACV ${ }^{14}$ (Tabela 2). Aqueles que já assumiram o estrato de alto risco após a análise do prontuário não foram entrevistados visto que não poderiam assumir um estrato superior a esse. Doze pacientes não puderam ser entrevistados por apresentarem rebaixamento significativo do nível de consciência e estarem sem acompanhante ou responsável.

A utilização da profilaxia mecânica para TVP foi avaliada com base nas recomendações da $\mathrm{SBACV}^{14}$. A fisioterapia é recomendada em todos os estratos de risco e seus métodos profiláticos consistem em cinesioterapia para membros inferiores ${ }^{15}$, deambulação precoce, compressão pneumática intermitente ${ }^{16,17}$, elevação de membros inferiores, uso de bandagens e meias elásticas e padrão ventilatório com inspiração máxima sustenta$\mathrm{da}^{18}$. Uma breve definição de cada método pode ser encontrada na Tabela 3.

O estudo estatístico foi realizado através do software SPSS versão 15.0, onde foi utilizado o teste QuiQuadrado, considerando o valor de $\mathrm{p}<0,05$.

\section{Resultados}

Foram analisados 282 pacientes, sendo 181 (64\%) homens e 101 (36\%) mulheres, com idade média de 54,1 anos (DP = 19,42). Quanto à estratificação do risco, 210 $(74,5 \%)$ foram classificados com alto risco, $56(19,8 \%)$ como moderado risco e $16(5,7 \%)$ como baixo risco. Do total de pacientes, $234(83 \%)$ não receberam profilaxia e $48(17 \%)$ receberam. Não houve diferença estatística significante entre os dados obtidos na pesquisa e os encontrados na literatura $(\mathrm{p}=0,065)$.

Dos 210 pacientes estratificados como alto risco, apenas 44 (21\%) receberam algum tipo de profilaxia mecânica, enquanto que dos 56 pacientes estratificados como médio risco, apenas $4(7,1 \%)$ receberam. Nenhum dos 16 pacientes estratificados como baixo risco recebeu profilaxia (Figura 1).

O método profilático mais utilizado foi a cinesioterapia para membros inferiores em 46 pacientes $(95,8 \%)$, 
Tabela 1 - Fatores de risco para trombose venosa profunda

\begin{tabular}{|c|c|c|c|}
\hline & Fatores clínicos & Medicamentos & Fatores cirúrgicos \\
\hline Idade & DPOC & Anticoncepcional oral & Procedimento cirúrgico \\
\hline Sexo & Insuficiência cardíaca & Reposição hormonal & Tempo da cirurgia \\
\hline \multirow[t]{16}{*}{ Dias de internação } & Insuficiência renal & Corticosteróides & Tipo de anestesia \\
\hline & Insuficiência hepática & & \\
\hline & AVE & & \\
\hline & Diabetes & & \\
\hline & Síndrome nefrótica & & \\
\hline & Infarto agudo do miocárdio & & \\
\hline & Arritmias & & \\
\hline & $\begin{array}{l}\text { Doença inflamatória dos } \\
\text { cólons }\end{array}$ & & \\
\hline & Obesidade & & \\
\hline & Imobilização & & \\
\hline & Doença auto-imune & & \\
\hline & Varizes & & \\
\hline & Desidratação & & \\
\hline & TVP prévia & & \\
\hline & Neoplasia & & \\
\hline & Infecção & & \\
\hline
\end{tabular}

AVE = acidente vascular encefálico; DPOC = doença pulmonar obstrutiva crônica; TVP = trombose venosa profunda. Fonte: Adaptado de Caiafa ${ }^{17}$.

seguido da deambulação precoce que foi utilizada em apenas 2 pacientes $(4,2 \%)$, como demonstrado na Figura 2. Os demais métodos profiláticos não foram utilizados.

\section{Discussão}

A incidência anual de tromboembolismo pulmonar (TEP) é estimada em 600 mil casos por ano nos EUA sendo um terço destes fatal, com metade dos óbitos ocorrendo na primeira hora após o início dos sintomas. A embolia está associada em 90\% dos casos a uma trombose no sistema venoso profundo dos membros inferiores, principalmente nas veias das panturrilhas ${ }^{19}$.

Já se encontra bem estabelecido que a TVP possui prevenção e que esta é eficiente na maior parte dos casos. É provável que a redução da mortalidade nas primeiras horas do TEP só seja possível por meio da prevenção, uma vez que não há tempo para medidas diagnósticas e terapêuticas de sucesso ${ }^{20}$. No entanto, o TEP continua uma constante causa de morte súbita em leitos hospitalares ${ }^{7}$.

Em um estudo realizado por Marchi et al..$^{5}$, apenas $20,45 \%$ dos pacientes receberam a profilaxia estudada, apresentando resultados similares a outros estudos feitos por Engelhorn et al. ${ }^{9}$ e Caiafa \& Bastos $^{21}$. Em países desenvolvidos a realidade é um pouco melhor, mas ainda insatisfatória como demonstram Goldhaber \& Tapson $^{22}$ em um estudo no qual dos 2.726 pacientes com diagnóstico de TVP ainda em seu período de permanência hospitalar, apenas $1.147(42 \%)$ haviam recebido profilaxia em um período de 30 dias que antecedeu o diagnóstico.

Em nossa pesquisa, do total de 282 pacientes com indicação para receber essa profilaxia, apenas $21 \%$ dos pacientes de alto risco e 7,1\% dos de médio risco receberam. A não prescrição da profilaxia mecânica para os 
Tabela 2 - Categorias de risco para doença tromboembólica

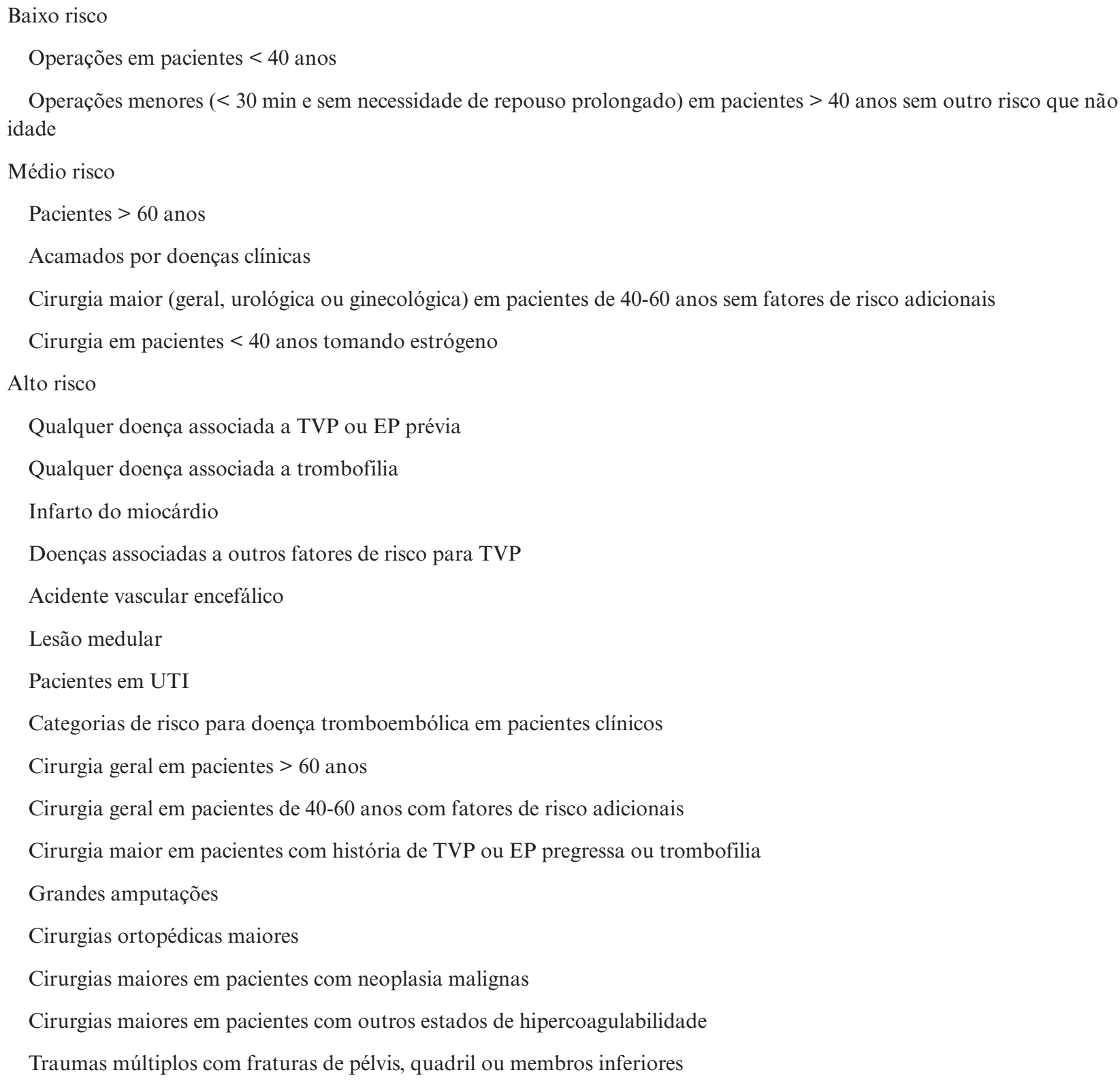

pacientes internados é um dos pontos que podem explicar a subutilização desse meio profilático, que só é realizado quando prescrito. É importante destacar que a profilaxia mecânica é um método bastante indicado para aqueles pacientes com risco de sangramento que pode ser causado pelo uso de anticoagulante, além de se tratar de uma prevenção eficaz e de baixo custo.
No ENDORSE ${ }^{23}$, um estudo observacional multinacional, apenas metade dos 68.183 pacientes receberam os métodos profiláticos recomendados (segundo o American College of Chest Physicians - ACCP). Dos pacientes cirúrgicos e clínicos com risco para TVP, 10\% foram classificados como sendo de alto risco para sangramento. A baixa utilização da profilaxia não pode ser 
Tabela 3 - Métodos de profilaxia fisioterapêutica

Cinesioterapia para membros inferiores

Deambulação precoce

Compressão pneumática intermitente

Elevação de membros inferiores

Bandagens e meias elásticas

Padrão ventilatório com inspiração máxima sustentada
É a realização de exercícios que produzem a mobilização articular, principalmente da articulação do tornozelo com intuito de ativar a bomba muscular da panturrilha, que ao contrair promove a compressão das veias diminuindo assim a estase venosa.

Promoção da estimulação natural e eficiente da bomba muscular da panturrilha, proporcionando a ejeção do sangue, pela ação conjunta da almofada do calcanhar potencializando o retorno venoso, diminuindo assim a probabilidade da incidência de TVP.

Compressão pneumática externa intermitente realizada por botas ou perneiras infladas cíclicas e seqüencialmente com ar com o objetivo de aumentar o fluxo venoso, principalmente quando aplicada compressão seqüencial tornozelo + panturrilha, tendo um aumento de $175 \%$ do fluxo do retorno venoso quando utilizada nas pressões entre 35 a $55 \mathrm{mmHg}$.

É um meio profilático simples e de baixo custo, que junto com a força da gravidade favorece o retorno venoso pela drenagem passiva do líquido acumulado nos MMII.

Atuam como compressão externa sobre os músculos e conseqüentemente sobre os vasos, auxiliando a dinâmica do fluxo de forma centrípeta, contrabalançando a insuficiência das válvulas e diminuindo a estase venosa.

Consiste em fazer com que o paciente, por meio do uso de inspirômetros incentivadores especialmente projetados, faça um trabalho ventilatório caracterizado por uma inspiração ativa forçada que deverá ser sustentada por um determinado intervalo de tempo mensurados em segundos em qualquer etapa da atividade terapêutica.

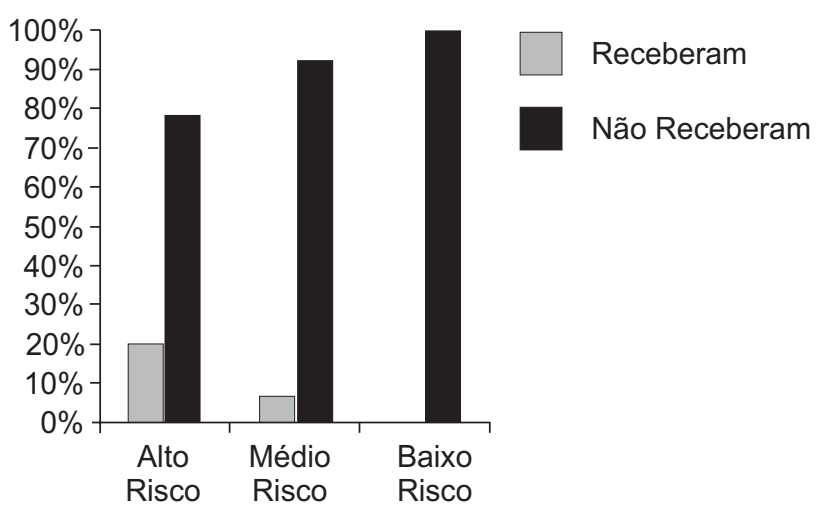

Figura 1 - Utilização da profilaxia mecânica

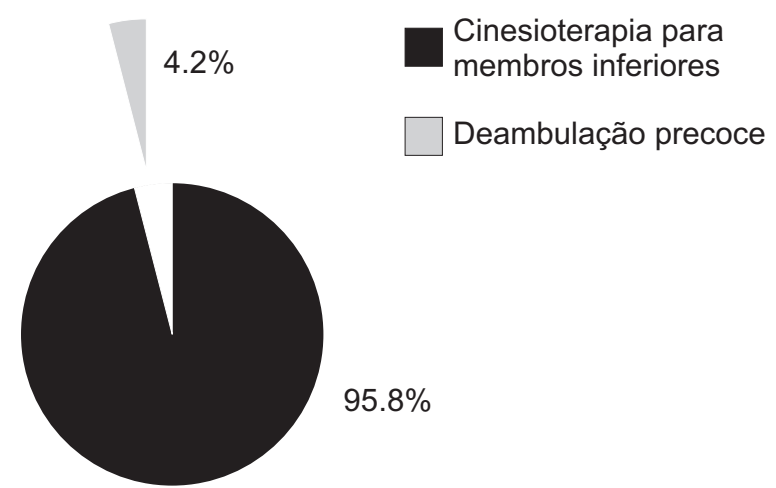

Figura 2 - Métodos profiláticos utilizados 
explicada unicamente por essa razão, pois esses pacientes poderiam ter recebido as formas de profilaxia mecânica. Entretanto, desses pacientes com contra-indicação para profilaxia anticoagulante, apenas 15\% receberam compressão pneumática intermitente isolada e $8 \%$ receberam meias de compressão graduada como o único método profilático.

Uma possível explicação para o elevado número de pacientes com alto risco de desenvolvimento de TVP em nosso estudo é a grande demanda de vítimas de acidente vascular encefálico-AVE (21\% do total de pacientes) e em menor proporção o número de vítimas de traumatismos medulares ( $6 \%$ do total), por se tratar de um grupo específico já classificado como de alto risco segundo o protocolo utilizado ${ }^{14}$. Nos casos de insuficiência cardíaca congestiva (ICC) descompensada, pode haver o receio do profissional fisioterapeuta em utilizar os métodos profiláticos, mesmo quando prescritos, devido o aumento da sobrecarga cardíaca. Porém, deve-se evitar a imobilidade física, fator de risco para TVP, podendo-se utilizar a cinesioterapia para membros inferiores cautelosamente ${ }^{24}$.

A falta de conhecimento sobre a profilaxia mecânica, dos protocolos de classificação quanto ao risco de TVP e a exigência de profilaxia medicamentosa prévia para os pacientes de médio e alto risco (segundo o protocolo utilizado) ${ }^{14}$ podem ser outras razões para a não utilização rotineira dessa profilaxia. Visando a mudança dessa realidade, é de extrema necessidade a implementação de estratégias educacionais com o objetivo de alertar os profissionais para a importância da profilaxia da TVP e quanto à gravidade e prevalência do tromboembolismo.

Alguns estudos já foram realizados com esses objetivos. Anderson et al. ${ }^{25}$ relatou um aumento no uso da profilaxia de $29 \%$ para $52 \%$ em pacientes hospitalizados com risco importante para desenvolvimento da trombose venosa, após a introdução de estratégias educacionais; o conhecimento dos dados estatísticos da doença tromboembólica no hospital onde trabalhavam foi um fator importante na adesão dos profissionais à profilaxia.
Já Maffei et al. ${ }^{26}$ observou o efeito da implementação de uma diretriz para a profilaxia do tromboembolismo venoso em um hospital privado, abrangendo médicos, enfermeiros e fisioterapeutas; embora tenha aumentado a preocupação com a profilaxia, aumentou pouco sua qualidade e tempo de utilização, sendo necessárias outras intervenções mais ativas e contínuas.

\section{Conclusão}

Com base nos dados apresentados, os autores concluem que apesar de sua eficácia já ter sido comprovada e difundida em diversos estudos, a profilaxia mecânica para trombose venosa profunda ainda é subutilizada na rotina hospitalar em questão, visto que só foi realizada em um pequeno número de pacientes com riscos potencias para o desenvolvimento da doença.

Esses dados demonstram a necessidade da implementação de estratégias de educação continuada para que os profissionais conheçam como intervir na prevenção da TVP e de suas complicações, quando prescrita a profilaxia. É imprescindível que toda a equipe multidisciplinar conheça os benefícios dessa profilaxia para saber quando indicar e prescrevê-las, visando uma melhor qualidade de vida do paciente e um custo-efetividade mais vantajoso para o serviço hospitalar.

\section{Referências}

1. Franco RM, Simezo V, Bortoleti RR, et al. Profilaxia para tromboembolismo venoso em um hospital de ensino. J Vasc Bras. 2006;5(2):131-8.

2. Maffei FHA, Rollo HA. Doenças vasculares periféricas. profunda dos membros inferiores: incidência, patologia, patogenia, fisiopatologia e diagnóstico. In: Maffei FHA, Lastódia S, Yoshida WB, Rollo HA. Doenças vasculares periféricas. $3^{\mathrm{a}}$ ed. Rio de Janeiro: Medsi; 2002. p. 1363-70.

3. Dryjski M, O'Brien-Irr MS, Harris LM, Hassett J, Janicke D. Evaluation of screening protocol to exclude the diagnosis of deep venous thrombosis among emergency department patients. J Vasc Surg. 2001;34:1010-5.

4. Silva MC. Epidemiologia do tromboembolismo venoso [editorial]. J Vasc Bras. 2002;1:83-4.

5. Marchi C, Schlup IB, Lima CA, Schlup HA. Avaliação da profilaxia da trombose venosa profunda em um hospital geral. J Vasc Bras. 2005;4:171-5.

6. Nicolaides AN, Breddin HK, Fareed J, et al. Prevention of venous thromboembolism: International Consensus Statement. Guidelines compiled in accordance with the scientific evidence. Int Angiol. 2001;20:1-37.

7. Clagett GP, Anderson FA Jr, Geerts W, et al. Prevention of venous thromboembolism. Chest. 1998;114(Suppl 5):531S-60S. 
8. Garcia ACF, Souza BV, Volpato DE, Deboni LM, Souza MV, Martinelli R, Gechele S. Realidade do uso da profilaxia para trombose venosa profunda: da teoria à prática. J Vasc Bras 2005;4:35-41.

9. Engelhorn ALV, Garcia ACF, Cassou AF, Birckholz L, Engelhorn CA. Profilaxia da trombose venosa profunda estudo epidemiológico em um hospital escola. J Vasc Bras. 2002;1:91-102.

10. Pitta GBB, Leite TL, Silva MDC, Melo CFL, Calheiros GA. Avaliação da utilização de profilaxia da trombose venosa profunda em um hospital escola. J Vasc Bras. 2007;6:344-51.

11. Anand SS, Wells PS, Hunt D, Brill-Edwards P, Cook D, Ginsberg JS. Does this patient have deep vein thrombosis? JAMA. 1998 8;279:1094-9.

12. Maffei FHA. Doenças vasculares periféricas. Profilaxia da trombose venosa e da embolia pulmonar. In: Maffei FHA, Lastódia S, Yoshida WB, Rollo HA. Doenças vasculares periféricas. $3^{\text {a }}$ ed. Rio de Janeiro: Medsi; 2002. p. 1487-98.

13. Hull RD. Doença venosa periférica. In: Goldman L, Bennet JC. Cecil: tratado de medicina interna. $21^{\mathrm{a}}$ ed. Rio de Janeiro: Guanabara Koogan; 2001. vol. 1, p.406-11.

14. Maffei FHA, Caiafa JS, Ramacciotti E, Castro AA para o Grupo de Elaboração de Normas de Orientação Clínica em Trombose Venosa Profunda da SBACV. Normas de orientação clínica para prevenção, diagnóstico e tratamento da trombose venosa profunda (revisão 2005). Salvador: SBACV; 2005. Disponível em: http://www.sbacv-nac.org.br

15. Kisner C, Colby LA. Exercícios terapêuticos. São Paulo: Manole; 2002. p.715-7.

16. Nicolaides AN, Fernandes e Fernandes J, Pollock AV. Intermittent sequential pneumatic compression of the legs in the prevention of venous stasis and postoperative deep venous thrombosis. Surgery. 1980;87:69-76.

17. Caiafa JS. Medidas profiláticas da doença tromboembólica. In: Thomás JB. Síndromes venosas: diagnóstico e tratamento. Rio de Janeiro: Revinter; 2001. p.195-208.

18. Azeredo CA. Fisioterapia respiratória no hospital geral. São Paulo: Manole; 2000. p. 225-41.
19. Stefanini E, Kasinski N, Carvalho AC. Guias de medicina ambulatorial e hospitalar de cardiologia. Cardiologia. São Paulo: Manole; 2004.

20. Pires MTB, Starling SV. Manual de urgências em Pronto Socorro. Rio de Janeiro: Guanabara Koogan; 2006.

21. Caiafa JS, Bastos, M de. Programa de profilaxia do tromboembolismo venoso do hospital naval Marcílio Dias: um modelo de educação continuada. J Vasc Bras. 2002;1:103-12.

22. Goldhaber SZ, Tapson VF; DVT FREE Steering Committee. A prospective registry of 5,451 patients with ultrasound-confirmed deep vein thrombosis. Am J Cardiol. 2004; 93:259-62.

23. Cohen AT, Tapson VF, Bergmann JF, et al. Venous thromboembolism risk and prophylaxis in the acute hospital care setting (ENDORSE study): a multinational cross-sectional study. Lancet. 2008; 371:387-94.

24. Regenga MM. Fisioterapia em cardiologia: da UTI à reabilitação. São Paulo: Roca; 2000.

25. Anderson FA Jr, Wheeler HB, Goldberg RJ, Hosmer DW, Forcier A, Patwardhan NA. Changing clinical practice. Prospective study of the impact of continuing medical education and quality assurance programs on use of prophylaxis for venous thromboembolism. Arch Intern Med. 1994;154:669-77.

26. Maffei FHA, Sato AC, Torggler Filho F, Silva SC, Atallah A. Efeito da implementação de diretrizes para profilaxia de tromboembolismo venoso em um hospital privado terciário. J Vasc Bras 2007; 6:105.

Correspondência:

Nathalia Leilane Berto Machado

Rua Xapuri, Conjunto Eldorado, 138, Bairro Feitosa

CEP 57043-470 - Maceió, AL

Tel.: (82) 3350.3281, (82) 8812.1761

E-mail: leilanemachado@hotmail.com 